\title{
VARIABILITY OF SURFACE FLOWS ON THE SUN AND THE IMPLICATIONS FOR EXOPLANET DETECTION
}

\author{
VALERI V. MAKAROV \\ NASA Exoplanet Science Institute, Caltech, 770 S. Wilson Ave., MS 100-22, Pasadena, CA 91125, USA; vvm@ caltech.edu \\ Received 2010 February 14; accepted 2010 April 6; published 2010 April 29
}

\begin{abstract}
The published Mount Wilson Doppler-shift measurements of the solar velocity field taken in 1967-1982 are revisited with a more accurate model, which includes two terms representing the meridional flow and three terms corresponding to the convective limb shift. Integration of the recomputed data over the visible hemisphere reveals significant variability of the net radial velocity at characteristic time scales of 0.1-10 years, with a standard deviation of $1.4 \mathrm{~m} \mathrm{~s}^{-1}$. This result is supported by independent published observations. The implications for exoplanet detection include reduced sensitivity of the Doppler method to Earth-like planets in the habitable zone, and an elevated probability of false detections at periods of a few to several years.
\end{abstract}

Key words: planets and satellites: detection - stars: individual (55 Cnc, HD 40307) - Sun: activity - techniques: radial velocities

\section{INTRODUCTION}

The spectacular success of the exoplanet search program, resulting in the discovery of over 300 planets and planetary systems to date, has been achieved mostly through the Dopplershift technique. Indeed, spectroscopic detections constitute the majority of known systems (Butler et al. 2006; Udry \& Santos 2007), with the bias toward short-period, massive planets probably due to the selection effects of this method. Undoubtedly, there is much room for further progress with the Doppler technique (Eggenberger \& Udry 2010), with the accuracy of spectroscopic instruments steadily improving and now reaching $\sim 1 \mathrm{~m} \mathrm{~s}^{-1}$, and the sensitivity of telescopes extending toward fainter stars. Combining precision photometric observations with radial velocity (RV) measurements provides a range of important physical characteristics of transiting stars invaluable for understanding the physics and the origin of exoplanets. The strategic goal of detecting rocky, habitable planets outside the solar system now seems to be coming within reach. As the instrumental precision steadily improves, growing attention has been paid to the intrinsic perturbations in the observable parameters used in exoplanet detection. Stochastic, uncorrelated physical perturbations increase the level of noise, making it difficult to achieve the threshold signal-to-noise ratio, while possible cyclic processes in the host stars can mimic exoplanet signatures. In particular, the rotating pattern of photospheric spots (Saar \& Donahue 1997) and irregularities in the convective structure on the surface (Meunier et al. 2010) can lead to an intrinsic scatter of RVs of up to a few $\mathrm{m} \mathrm{s}^{-1}$ for solar-type $\mathrm{G}$ dwarfs. In the younger Hyades, which are more magnetically active and rotate faster than the Sun, this effect is magnified to $\sim 16 \mathrm{~m} \mathrm{~s}^{-1}$ in standard deviation (STD), as observed by Paulson et al. (2004). As the level of activity is not constant in solar-type stars, the intrinsic RV scatter is correlated with the magnetic cycle, opening possibilities of more sophisticated spectroscopic analysis in order to mitigate these difficulties. For example, the index of chromospheric activity is correlated with the area of star spots (and hence, with the observed scatter) and can serve as an indicator of magnetically induced cycles (Santos et al. 2000). Careful selection of target stars can further improve the prospect of detection of smaller planets with the Doppler technique. Recent simultaneous measurements of chromospheric activity and $\mathrm{RV}$ imply that $K$-type dwarfs may be significantly less variable than the Sun, and the intrinsic RV jitter is less than $1 \mathrm{~m} \mathrm{~s}^{-1}$ (Santos et al. 2010). Some stars older than 6 Gyr and evolved off the main sequence have sharply reduced levels of magnetic activity (Wright 2004) compared to the Sun. In this paper, we consider another important source of intrinsic RV variation, related to the physical motion of the surface layers of stars, which remained largely outside the scope of previous papers.

The surface layers of the Sun, where the spectroscopic lines are formed, are known to be involved in a complex pattern of radial and tangential motion. Furthermore, it is now an observational fact that this velocity field is not static. In this paper, the series of Doppler measurements taken at the Mount Wilson Observatory is revisited, and the published fitting model coefficients are transformed to a different model of differential rotation, convective blueshift, and meridional flow, which may more adequately represent the reality (Section 2). The transformed model is integrated to produce the net $\mathrm{RV}$ of the Sun as a star (Section 3). These results are compared to other data on the variability of the main components of the velocity field in Section 4, and a good agreement is found. The impact of the intrinsic variation of solar RV on the detectability of exoplanets, especially within the habitable zone, is investigated in Section 5 by means of $\chi^{2}$ periodograms as well as by a planet detection experiment, which results in two bogus planets. The relative importance of surface flows compared with other sources of intrinsic RV perturbations is discussed in Section 6.

\section{MOUNT WILSON DATA}

An extensive set of Doppler velocity measurements taken at Mount Wilson during a period of 14 years, from 1967 through 1982, was published by Howard et al. (1982). The full-disk magnetograms in Fe I line $5250.2 \AA$ were fitted with the model ${ }^{1}$

$$
\begin{aligned}
V= & \left(A+B \sin ^{2} \phi+C \sin ^{4} \phi\right) \sin \lambda \cos \phi \cos b_{0} \\
& +\left(D+E \sin ^{2} \phi+F \sin ^{4} \phi\right) \sin ^{2} \lambda \cos \phi \cos b_{0}+G
\end{aligned}
$$

with $\phi$ the solar latitude, $\lambda$ the longitude (heliocentric angle from the central meridian), $b_{0}$ the solar latitude of disk center, $G$ an arbitrary zero-point velocity, and $A, B, C, D, E$, and $F$ free fitting parameters. The first group of terms with coefficients $A$,

\footnotetext{
The instrumental drift term has been omitted.
} 
$B$, and $C$ represents differential rotation, with $A$ denoting the equatorial velocity. Since the terms of differential rotation are odd functions of $\lambda$, their net contribution to the integral RV is zero, and we are not concerned with them in this paper. The second group of terms with $D, E$, and $F$ can be interpreted as the antisymmetric component of the differential rotation. These terms are even in $\lambda$ and result in a net contribution to the RV. However, because the observations are differential and the term $G$ is arbitrary, the total offset of velocity is indeterminate, and only the variation of these terms in time can be considered.

The integral RV as measured by a distant observer is

$$
\begin{aligned}
\bar{V}= & \frac{1}{2 \pi} \int_{-\frac{\pi}{2}}^{\frac{\pi}{2}} d \lambda \int_{-\frac{\pi}{2}}^{\frac{\pi}{2}}\left(\alpha_{0}+\alpha_{1} \cos \phi \cos \lambda+\alpha_{2} \cos ^{2} \phi \cos ^{2} \lambda\right) \\
& \times V \cos \phi \cos \lambda \cos \phi d \phi .
\end{aligned}
$$

In this formula, $\alpha_{0}, \alpha_{1}$, and $\alpha_{2}$ are the coefficients of solar limb darkening. Adopting $\alpha_{0}=0.30, \alpha_{1}=0.93$, and $\alpha_{3}=-0.23$, the resulting net $\mathrm{RV}$ is

$$
\bar{V}=0.1005 D+0.01841 E+0.007417 F .
$$

Using the tabulated data in Howard et al. (1982), we compute an RV curve, which shows significant variations of $4.95 \mathrm{~m} \mathrm{~s}^{-1}$ in STD. However, this result is likely overestimated for the following reasons.

As was pointed out by Snodgrass \& Ulrich (1990) and Snodgrass \& Howard (1985), the terms of differential rotation $A, B \sin ^{2} \phi$, and $C \sin ^{4} \phi$ are not orthogonal on a halfsphere, resulting in considerable correlations between the coefficients determined from regularly sampled data. They suggested to use Gegenbauer polynomials in $\sin \phi$ to orthogonalize the terms. The first thus orthogonalized coefficient corresponding to the principal component of solar rotation is $\hat{A}=$ $A+B / 5+3 C / 35$. Note that the coefficients in this relation are closely in the same proportion (1:0.2:0.085) as the coefficients in Equation (3), (0.1005:0.01841:0.007417). This coincidence may be interpreted as a real net variation of the integral velocity (which cannot be captured by the odd $A, B$, and $C$ terms) translating into the non-orthogonal $D, E$, and $F$ terms according to their covariances, since all the differential measurements are brought to a single zero point. This hypothesis is supported by the actual negative correlation of the three terms in Equation (3), whose variances add up to a larger number than $4.95^{2}$.

Thus, a real variation of the surface velocity field was probably exaggerated in the model (1) because the $D, E$, and $F$ terms are internally correlated. Besides, these terms poorly describe the real velocity field. Indeed, the functional form of $D, E$, and $F$ terms corresponds to a pattern of zonal stretches on the surface, which remain fixed with respect to the central meridian despite the general rotation of the Sun. Clearly, any longitudinal perturbations of rotation velocity cannot remain static with respect to the observer on time scales longer than one rotation period. The correct physical explanation of the symmetric pattern of Doppler velocities observed at Mount Wilson was given by LaBonte \& Howard (1982). The mysterious looking zonal pattern of RV is caused by two separate physical phenomena on the Sun: the axisymmetric convective blueshift (often called the limb shift) and the meridional surface flow symmetric around the equator to first approximation.

The model of meridional flow considered by LaBonte \& Howard (1982) assumed a constant surface velocity directed toward the poles. We replace it with a more adequate model suggested by Hathaway et al. (1996):

$$
V_{\mathrm{M}}=\mu_{2} P_{2}^{1}(\sin \phi)+\mu_{4} P_{4}^{1}(\sin \phi),
$$

where $P_{l}^{1}$ are associated Legendre polynomials of degree $l$ and order 1 . The corresponding tangential velocity field is depicted in Figure 1. The free fitting parameters $\mu_{2}$ and $\mu_{4}$ are to be determined from observations. The Legendre polynomials are orthogonal on the range of $\sin \phi$, which drastically simplifies the subsequent analysis. We also replace a polynomial expansion for limb shift with the more sophisticated model from (Hathaway 1996)

$$
V_{\mathrm{B}}=\sum_{k=1}^{3} \beta_{i} P_{k}^{*}(1-\cos \rho),
$$

where $P_{k}^{*}$ are shifted Legendre polynomials orthogonal on $[0,1], \rho$ is the central angle $(\cos \rho=\cos \phi \cos \lambda)$, and $\beta_{i}$ are free fitting coefficients.

Thus, the new model which more faithfully represents the observed velocity field is

$$
\begin{aligned}
V= & \left(A+B \sin ^{2} \phi+C \sin ^{4} \phi\right) \sin \lambda \cos \phi \cos b_{0} \\
& +\left(\mu_{2} P_{2}^{1}(\sin \phi)+\mu_{4} P_{4}^{1}(\sin \phi)\right) \\
& \times\left(\cos \lambda \sin \phi \cos b_{0}-\cos \phi \sin b_{0}\right)+V_{\mathrm{B}}+G .
\end{aligned}
$$

We carried over the $A, B$, and $C$ terms of differential rotation (rather than using Gegenbauer polynomials) to simplify subsequent transformations.

Our task is now to recompute the observed parameters of the velocity field using the published $A, B, C, D, E$, and $F$ parameters. The fitting procedure is a linear least-squares problem and the transformation problem can be expressed in matrix notation

$$
[\Lambda \mathbf{\Phi}] \tilde{\mathbf{x}}=[\boldsymbol{\Lambda} \mathbf{T}] \overline{\mathbf{x}}+\epsilon,
$$

where $\boldsymbol{\Lambda}$ is the matrix of differential rotation (the $A, B$, and $C$ terms), $\Phi$ is the matrix of the symmetric stretch (the $D, E$, and $F$ terms), $\mathbf{T}$ is the matrix of the new meridional flow and limb shift terms, $\tilde{\mathbf{x}}$ is the vector of free parameters of the old model, $\overline{\mathbf{x}}$ is the vector of free parameters of the new model, and $\epsilon$ is random noise. It can be shown that in a least-squares solution,

$$
\overline{\mathbf{x}}=\mathbf{T}^{\dagger}[\boldsymbol{\Lambda} \Phi] \tilde{\mathbf{x}},
$$

where $\mathbf{T}^{\dagger}=\left(\mathbf{T}^{\mathrm{T}} \mathbf{T}\right)^{-1} \mathbf{T}^{\mathrm{T}}$ is the pseudoinverse of $\mathbf{T}$. The matrix and inner vector products here are calculated through integration of the element products over the visible disk with the $\cos \phi$ weight. The inner products of the new model terms and the $A$, $B$, and $C$ terms are all zeros (in other terms, these functions are orthogonal). Therefore, the $A, B$, and $C$ coefficients remain unchanged in this transformation, and the new parameters depend only on the $D, E$, and $F$ coefficients.

After some toil with integration and matrix inversion, one obtains

$$
\left[\begin{array}{l}
\mu_{2} \\
\mu_{4} \\
\beta_{1} \\
\beta_{2} \\
\beta_{3}
\end{array}\right]=\left[\begin{array}{rrr}
-0.766 & -0.252 & -0.119 \\
0.482 & 0.094 & 0.018 \\
0.345 & 0.108 & 0.053 \\
0.012 & 0.030 & 0.026 \\
-0.106 & -0.030 & -0.010
\end{array}\right] \cdot\left[\begin{array}{l}
D \\
E \\
F
\end{array}\right] .
$$




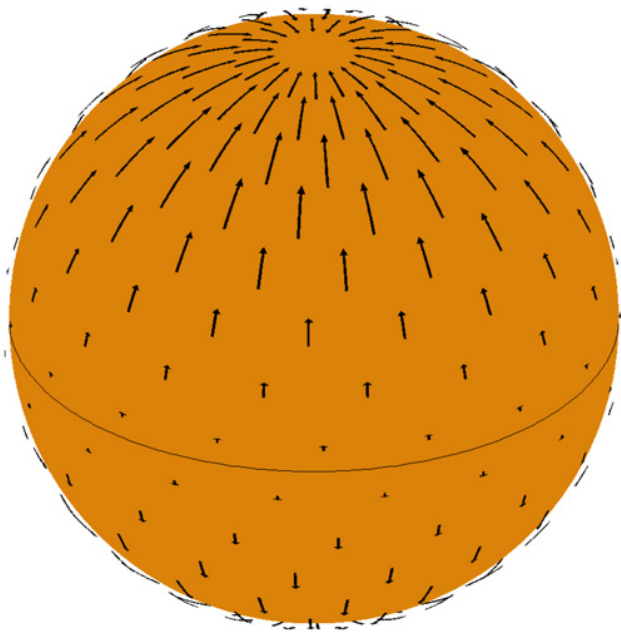

Figure 1. Velocity field of solar meridional flow. The net motion is directed from the equator toward the poles, vanishing at the equator and the poles.

\section{THE SOLAR RV VARIATION}

Analogous to Equation (2), the new model of the solar velocity field can be integrated over the visible hemisphere to yield

$$
\bar{V}=-0.157 \mu_{2}+0.025 \mu_{4}-0.295 \beta_{1}+0.044 \beta_{2}+0.004 \beta_{3} .
$$

This result is valid only for the Sun seen edge-on (inclination $90^{\circ}$ ), although the limb shift is probably independent of inclination. The five terms of the new model are derived from only three terms of the old model, hence they are strongly correlated because of the projection in the function space. The reconstructed RV curve is shown in Figure 2. The original data in Howard et al. (1982) are sampled by Carrington rotations; therefore, each data point represents the mean over 27.2753 days. Monthly variations of a few $\mathrm{m} \mathrm{s}^{-1}$ are common, but so are longer-term variations on time scales of years. A running median shown with the red line indicates that the long-term RV of the Sun grew by $\simeq 7 \mathrm{~m} \mathrm{~s}^{-1}$ between 1976 and 1981 . The reconstructed $\mathrm{RV}$ curve has an STD of $1.44 \mathrm{~m} \mathrm{~s}^{-1}$. The long-term behavior of solar RV is strongly correlated with the magnetic cycle (cf. the monthly sunspot numbers in the lower panel of Figure 2). RV variations on time scales of several months to 1 year have a strong impact on the detectability of habitable planets around solar-type stars, as discussed in Section 5. We have to carefully investigate if these results are consistent with other independent data on the Sun.

\section{COMPARISON WITH OTHER DATA}

Noting that the low-order terms of meridional flow $\left(\mu_{2}\right)$ and limb shift $\left(\beta_{1}\right)$ propagate with the largest weights into the integral velocity in Equation (10), we have to consider what is known about the variability of these physical effects. The most direct comparison comes from the Doppler observations with the Global Oscillation Network Group (GONG). Hathaway et al. (1996) present the results for the main component of the meridional flow over some 130 days in 1995 (their Figure 1, top panel), corresponding to $P_{2}^{1}$ (Legendre polynomial of degree 2 and order 1). These coefficients are denoted $\mu_{2}$ in Equation (10). The flow velocity varies between 0 and $50 \mathrm{~m} \mathrm{~s}^{-1}$, which for an edge-on Sun translates to a peak-to-peak amplitude of $8 \mathrm{~m} \mathrm{~s}^{-1}$. Quasi-periodic cycles of 10-20 days are evident in the GONG data as well as longer-term variations on a few months, which are expected to change the integral velocity by roughly $3-4 \mathrm{~m} \mathrm{~s}^{-1}$. The amplitudes of the higher-degree terms $P_{4}^{1}$ and $P_{6}^{1}$ are found to be much smaller $(-1.6 \pm 0.5$ and $-1.3 \pm 0.1 \mathrm{~m} \mathrm{~s}^{-1}$ ), so their contribution to the net RV is negligible in the context of this paper.

On longer time scales, the main $l=2$ component of meridional circulation was investigated by Hathaway (1996), also based on the GONG Doppler measurements. The flow velocity grew from $\sim 20 \mathrm{~m} \mathrm{~s}^{-1}$ in the summer of 1992 to almost $100 \mathrm{~m} \mathrm{~s}^{-1}$ by the end of 1993 , then dropped to small negative values in the summer of 1994, indicating a brief episode of reversal. After that, it gradually returned to $\sim 20 \mathrm{~m} \mathrm{~s}^{-1}$ by mid1995. From Equation (10), the overall RV should have changed by $16 \mathrm{~m} \mathrm{~s}^{-1}$ in the first half of 1994. Unfortunately, our data in Figure 2 cover an earlier time interval and cannot be directly

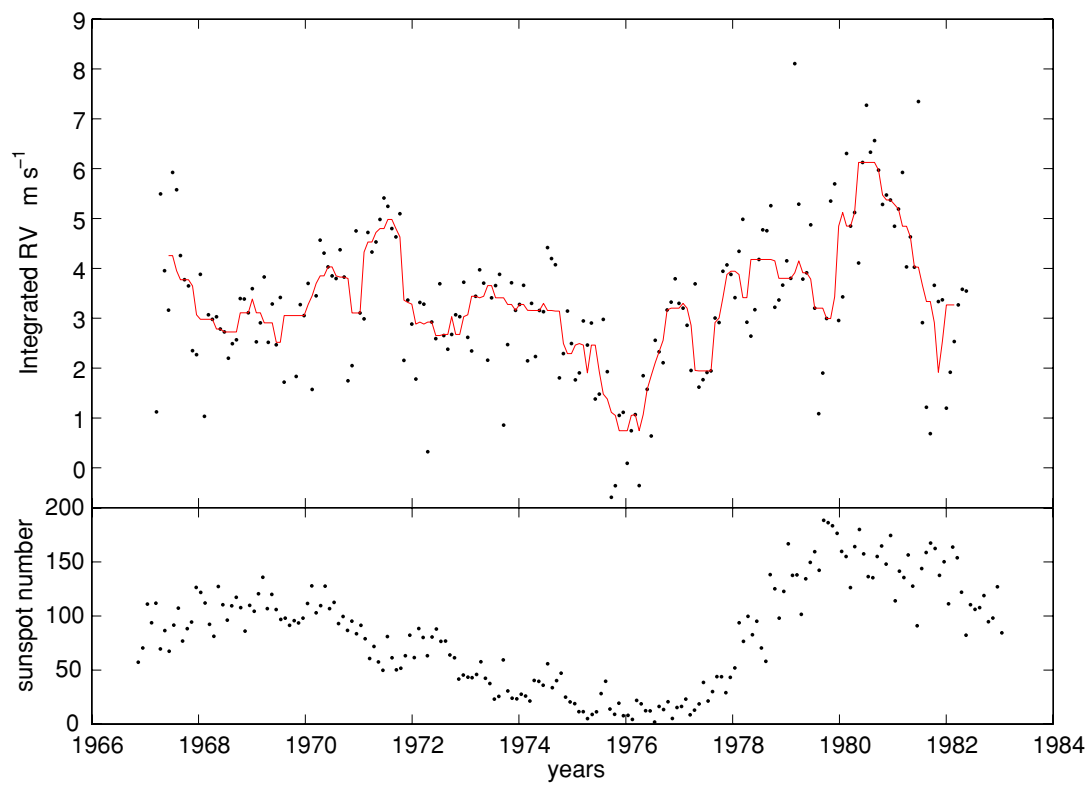

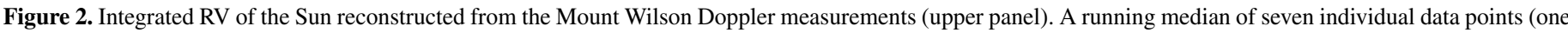

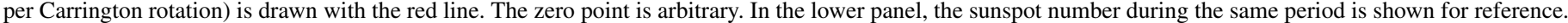


compared with the GONG results. But the scale of variability in our reconstruction is absolutely consistent with the newer and more accurate data.

In the same paper, Hathaway (1996) presents a reconstruction of the history of the three terms of convective blueshift, denoted $\beta_{1}, \beta_{2}$, and $\beta_{3}$ in this paper. Only the first term appears to be a significant contributor to the net velocity, with a quasi-sinusoidal variation of $\sim 30 \mathrm{~m} \mathrm{~s}^{-1}$ on a period of $1.5 \mathrm{yr}$ and a short-term scatter of similar magnitude. The corresponding range of $\mathrm{RV}$ is $0.295 \times 30 / \sqrt{3}=5 \mathrm{~m} \mathrm{~s}^{-1}$. Little correlation was noted between the meridional flow and the limb shift on the GONG data.

The meridional flow traced by the motion of magnetic features in the photosphere from a 26-year set of Mount Wilson magnetograms appears to be somewhat smaller in amplitude and more complex in the equatorial zone, with strong equatorward features (Snodgrass \& Dailey 1996). The maximum rate is $\sim 13 \mathrm{~m} \mathrm{~s}^{-1}$, and the pattern is strongly correlated with the solar cycle. A detailed picture of the solar cycle-related variation in the structure of the meridional flow is shown in Ulrich \& Boyden (2005, their Figure 2). The poleward components of the meridional circulation are the strongest when the solar activity is at its maximum, in agreement with the reconstructed data in Figure 2. Gizon \& Rempel (2008) used $\mathrm{SOHO}$ Michelson Doppler Imager (MDI) full-disk Doppler images for the period 1996-2002 and measured the advection of the supergranulation pattern, which is probably consistent with the tracing of magnetic features. They detect only poleward motions reaching $13 \mathrm{~m} \mathrm{~s}^{-1}$ in the antisymmetric part of the field averaged in $1 \mathrm{yr}$ intervals. The peak-to-peak variation of the antisymmetric component is $7 \mathrm{~m} \mathrm{~s}^{-1}$. This is again in agreement with the magnitude of $R V$ variation derived in this paper.

With the abundance of high-quality data, the analysis procedures are very complex, which account for some lingering disagreement between different published results on the solar velocity field. One of the most rigorous and comprehensive models was presented by Hathaway (1987), including differential rotation, torsional streams, meridional circulation, the limb shift, supergranules, and giant cells. The surface velocity field is represented with vector spherical harmonics, which are orthogonal on a unit sphere. Although this much desired orthogonality is compromised by the availability of only one hemisphere, inclination, and projection effects (as far as Doppler data are concerned), the various scales of poloidal and toroidal motions are naturally separated in the model through vector spherical harmonics of different degrees and orders. For example, the three main components of differential rotation are represented by the toroidal harmonics of zero-order $\mathbf{T}_{1}^{0}$ (solid body rotation), $\mathbf{T}_{3}^{0}$, and $\mathbf{T}_{5}^{0}$. The limb shift terms are correlated (non-orthogonal) to the meridional flow terms to such extent, that their separation requires a full-cycle iteration (Hathaway 1992), especially if the aim is to reconstruct the absolute velocity field. This seems to be the only way to infer the main term of limb shift (the value at the center of the disk), which is $-540 \mathrm{~m} \mathrm{~s}^{-1}$ according to Hathaway. Thus, the variable component of the limb shift is only $\approx 1 \%$ of the constant component.

Similarly, the large-scale meridional flows are dwarfed by the supergranulation pattern, which has typical velocities of $300-400 \mathrm{~m} \mathrm{~s}^{-1}$ (Hathaway et al. 2000). The high-degree supergranulation is of little interest in the context of this paper, because it only contributes to the high-frequency variation of the integral RV, whereas we are interested in what happens with solar RV on the time scales of months to years. But the lower-degree supercells are of interest, because they rotate

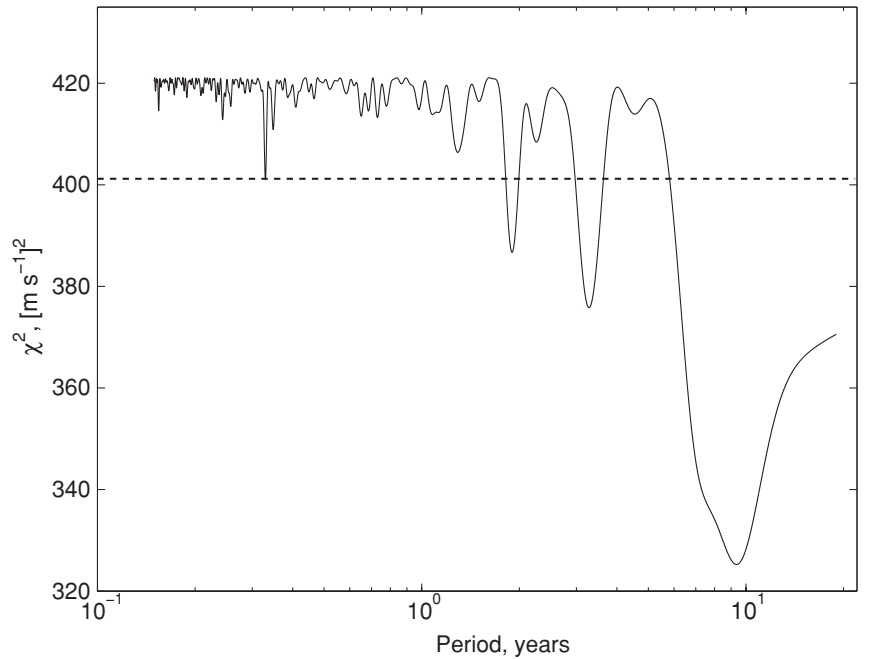

Figure 3. $\chi^{2}$-periodogram of the reconstructed solar RV curve in Figure 2. The dashed horizontal line indicates the threshold $\chi^{2}$ corresponding to a false alarm probability of $1 \%$ for the first planet detection.

with the Sun, indicating physical flows in the photosphere. A rotating spherical harmonic term of order $m$ will produce a periodic signal in the integral velocity with a period of $P_{\odot, \text { rot }} / \mathrm{m}$. The power spectrum of the velocity field peaks at degrees $l \sim 120$, which correspond to the spatial scale of supergranules. Therefore, rotating supergranules will cause a net variation in $\mathrm{RV}$ of a few $\mathrm{m} \mathrm{s}^{-1}$ on time scales of a few hours.

\section{THE IMPACT ON EXOPLANET DETECTION}

Exoplanets are found in Doppler measurement data through the periodic perturbations caused by the orbital motion of the host star around the system barycenter. To a good approximation, the RV of the star is the sum of a constant term (the velocity of the barycenter) and a number of Kepler motions. For loweccentricity orbits, such as those in the solar system, the Kepler velocity signal is almost perfectly sinusoidal. Detecting one or several sinusoidal variations in high-cadence, Doppler measurements superimposed with a constant offset is fairly straightforward, if the observational noise is random, uncorrelated, and sufficiently small compared to the expected signal.

An additional source of noise or perturbation from a real physical process on the star makes this detection significantly more difficult, or in some cases, ambiguous. To quantify the impact of the "surface flow noise" on the detectability of planets, a generic planet detection algorithm was applied to the data derived in Section 3. No additional noise was added to the individual RV points. The procedure starts with a periodogram analysis and a subsequent estimation of the confidence of the most prominent sinusoidal variations. Figure 3 shows the resulting periodogram of the reconstructed solar RVs. Instead of the traditional spectral power, the residual $\chi^{2}$ is plotted for a high-cadence grid of periods. The $\chi^{2}$-statistics are preferred, because the confidence level of the detected minima is rigorously computed via the cumulative probability function of the $F$ statistics with $\left\{n_{\mathrm{obs}}-3,2\right\}$ degrees of freedom. This confidence $F$-test is analogous to the estimation of false alarm probability (FAP) by Cumming (2004), with the number of independent frequencies corresponding to the frequency search interval. Each dip in this curve, which corresponds to a value of $F$ surpassing a given confidence threshold, can be considered 


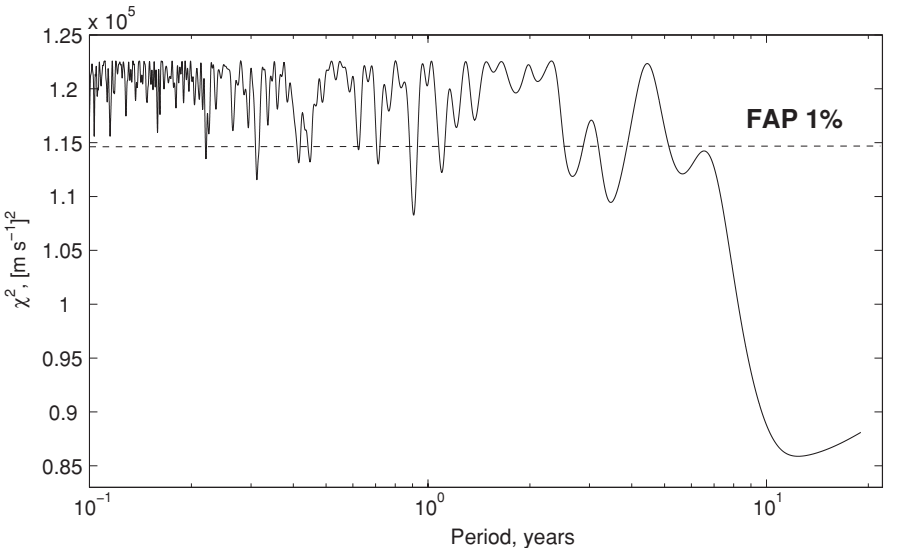

Figure 4. $\chi^{2}$-periodogram of the RV measurements of $55 \mathrm{Cnc}$, which were used to detect the five exoplanets reported in the literature. The dashed horizontal line indicates the threshold $\chi^{2}$ corresponding to a false alarm probability of $1 \%$ for the first planet detection.

a planet detection. Setting the confidence threshold at $99 \%$, this periodogram analysis results in the detection of two bogus planets with orbital periods $P_{1}=9.35 \mathrm{yr}$ (mass $26 M_{\oplus}$ ) and $P_{2}=6.35 \mathrm{yr}$ (mass $15 M_{\oplus}$ ). The former false detection is apparently triggered by the long-term variation of the meridional flow with the solar cycle, and the latter may also be related to some poorly understood cyclicity of magnetic activity, because a sinusoidal variation of similar frequency emerges in the Total Solar Irradiance (TSI) data.

This numerical experiment indicates that the magnetic cyclerelated variations in Sun-like stars can in some cases be confused with long-period planets. It is therefore of interest to compare the reconstructed solar periodogram with accurate RV data for a planet host. Figure 4 shows a $\chi^{2}$-periodogram for the star $55 \mathrm{Cnc}$ with high-resolution spectroscopic measurements from Marcy et al. (2002) and Fischer et al. (2008). The total span of these measurements is $18.3 \mathrm{yr}$, and the formal single measurement error progressed from $\sim 9 \mathrm{~m} \mathrm{~s}^{-1}$ during the first six years (Lick observations) to between 3 and $5 \mathrm{~m} \mathrm{~s}^{-1}$ over the later period (Keck observations). The star is a K0/G8 dwarf, slowly rotating and chromospherically inactive $\left(\log R_{\mathrm{HK}}^{\prime}=-4.84\right)$. It is a close, albeit somewhat smaller, solar analog. Five planets have been reported in the literature (Fischer et al. 2008), with orbital periods from 2.8 days to 14 years. Figure 4 depicts a portion of the $\chi^{2}$-periodogram computed from the weighted RV data for the same range of periods as in Figure 3. Our confidence estimation is consistent with the FAP thresholds computed in the cited papers, where a different technique was used (Monte Carlo trials on randomly scrambled data). Except for a number of pronounced high-frequency dips, some of which correspond to the detected planets, the structure of the periodogram is quite similar to that for the Sun. In particular, the lowest dip in this part of the periodogram centered on $14 \mathrm{yr}$ at the long-period end, corresponding to the planet $55 \mathrm{Cnc} \mathrm{d}$, is reminiscent of the 9.35-yr feature for the Sun, which is quite likely caused by the solar cycle. This similarity calls for caution in the interpretation of long-period signals from stars like $55 \mathrm{Cnc}$.

Extrapolating the observational results presented in Section 2 to other stars requires caution. The Mount Wilson data are based on a single Fe line, whereas an RV curve for exoplanet detection is derived from a large number of weak lines across the visible spectrum. There are good reasons to assume, however, that the dominating $\mu$-terms of the meridional flow in Equation (6) are not wavelength-dependent. The existing models of motions in the Sun (still somewhat uncertain) imply large-scale and deeprooted dynamical structures of global character. For example, Howard (1987) presented a model of only several gigantic longitudinal supercells (or rolls), which may evolve or drift with the magnetic cycle. In this interpretation, the large-scale meridional flows are quite deep. The case with convective limb shift, which is wavelength-dependent, is perhaps more complicated. The blueshift is not the same in different lines, but it seems plausible that the long-term changes due to the 11-yr and other known magnetic cycles are caused by a slower or faster convective motion on the integrated disk, thus resulting in a uniform shift of line bisectors across the spectrum. Systematic changes in line shapes can be expected due to magnetic cycles as well. Recently, Santos et al. (2010) investigated a sample of eight late-G or early-K dwarfs of low magnetic activity and compared precision RV measurements with simultaneous estimates of chromospheric activity. They could not find any clear correlation and concluded that any RV variations induced by magnetic cycles on these stars are unlikely to be much greater than $1 \mathrm{~m} \mathrm{~s}^{-1}$. Therefore, it is possible that many $\mathrm{K}$ dwarfs are less subject to the adverse effects of variable surface flows than more solar-like stars.

Most of the currently known or suspected exoplanets have masses of the solar system giants (or larger) and short-period orbits (Butler et al. 2006; Udry \& Santos 2007). A considerable effort is under way to improve the sensitivity of the Doppler instruments and data calibration to the levels sufficient to detect Earth-like, rocky planets within the habitable zones of mainsequence stars, where water can be present in the liquid state. The required single measurement error due to photon noise and instrument imperfection should be $\lesssim 0.1 \mathrm{~m} \mathrm{~s}^{-1}$ to achieve this goal. One should consider the possibility that the surface flow and convective blueshift perturbations are in fact much larger than $0.1 \mathrm{~m} \mathrm{~s}^{-1}$ in the range of habitable orbits too. Using the reconstructed solar RV curve in Figure 2, I computed the amplitude spectrum (i.e., the amplitude of the best-fitting sinusoid as a function of frequency) for a range of periods $0.5-1.5 \mathrm{yr}$, roughly corresponding to the solar habitable zone. The power density turned out to be quite flat at $\simeq 1 \mathrm{~m} \mathrm{~s}^{-1}$. This result only sets the upper bound for the actual power spectrum of RV variations of the Sun, since the contribution of observational error in the Mount Wilson data is not known. Ultra-precise, long-term RV observations of stable solar-type stars will be required to confirm that the intrinsic RV variations come up to such values. We note that a $3 \sigma$ detection of Earth in a periodogram requires the perturbation amplitude to be less than $0.03 \mathrm{~m} \mathrm{~s}^{-1}$.

\section{DISCUSSION}

Variable surface flows are only one of the known physical processes on the Sun that can change the integrated RV. They appear to be long term in nature, and therefore may be the main obstacle to detecting Earth-like habitable planets with ultraprecise Doppler measurements. The main pulsation modes of solar-type stars ( $p$-modes) have periods of 10-20 minutes and can be successfully suppressed in observations by taking longer exposures. The supergranulation pattern, discussed in Section 4, should produce variations of up to a few $\mathrm{m} \mathrm{s}^{-1}$ but their time scale is several hours. Rotation of stars and the non-uniform distribution of surface brightness due to photospheric spots and plages are probably the main source of RV perturbations on time scales up to 50 days. The impact of spots has been 
investigated in numerous papers, and the latest estimates suggest relatively small, but non-negligible, dispersion for the Sun of $\sigma_{\mathrm{RV}} \simeq 0.4 \mathrm{~m} \mathrm{~s}^{-1}$ (Makarov et al. 2009). As was shown by Paulson et al. (2004) for the Hyades, the photometric and the RV jitter of more active stars than the Sun are strongly correlated. Extrapolating the empirical relation found in that paper, one would expect a jitter of $\sigma_{\mathrm{RV}}=1.7 \mathrm{~m} \mathrm{~s}^{-1}$ for the Sun. The large discrepancy between these estimates is probably related to the different morphology of photospheric inhomogeneities in the Hyades stars. Dark spots are the dominating magnetic features on active and young stars, whereas the contribution of bright plages on older, solar-type stars tends to match the impact of spots, or even to prevail (Lockwood et al. 2007). Besides, active stars often have only one or two giant long-lived spots on the surface, resulting in a strong rotational modulation of the light curve, whereas the surface of an active Sun is usually marked with a few spot groups at a time, fairly uniformly distributed in longitude. Meunier et al. (2010) estimated the combined impact of sunspots and plages at $\simeq 1 \mathrm{~m} \mathrm{~s}^{-1}$ in RV amplitude, and concluded that it should be confined to periods less than 100 days. Our study suggests that on the time scales $0.6-1.4 \mathrm{yr}$, characteristic of orbits within the habitable zones, slowly evolving surface flows and the distribution of the convective blueshift is the major source of confusion and error.

Our conclusions are in agreement with the observations of solar RV by McMillan et al. (1993). These authors observed the solar light reflected from the Moon for 5 years nightly in violet absorption lines and determined an upper limit of $4 \mathrm{~m} \mathrm{~s}^{-1}$ for the overall dispersion of RVs. The periodogram of RV variations (their Figure 4) shows four peaks rising above the $3 \sigma$ threshold, all in the long-period part of the spectrum. Three of them may be of instrumental origin, but the unresolved peak at period longer than $3 \mathrm{yr}$ appears to be genuine and is called "intriguing" in the paper. The two bogus planets found in the reconstructed RV data in this paper (Section 5) have periods 6.35 and $9.35 \mathrm{yr}$. Therefore, it is possible that McMillan et al. (1993) already detected long-term variations in the RV of the Sun as a star. Meunier et al. (2010) pointed out that the effects of convective blueshift, which is included in our RV model (Section 2), may be smaller in the deep violet lines than in the redder lines that are normally used for planet search.

Our final note is that the model of meridional flow depicted in Figure 1 is a simplified representation of the actual fine structure of the velocity field. By necessity, the model for the Mount Wilson data is coarse, where the contribution of convective blueshift and meridional flow cannot be decoupled. The former is especially problematic, because it is wavelengthdependent, and uncalibrated changes in the spectrograph setup can bring about long-term systematic errors (cf. Ulrich 2001).
The low-order polynomials used to fit the surface velocity field (Section 2) only reveal the underlying, very much smoothed structure of the actual distribution of tangential and radial motion, characterized by smaller spatial scales and larger amplitudes. For example, the local flows are known to converge toward active regions, reaching 50-100 m s$~^{-1}$ (Gizon 2004), and these local inflows may be responsible for the temporal variation of the overall velocity field on the time scales of supergranulation $(8 \mathrm{hr})$ and the solar cycle (11 years). Thus, the temporal behavior of the velocity field may be in part the average of many stochastic components, and in part the evolution of the global magnetic field (Ulrich \& Boyden 2005), both still poorly understood for the Sun. Therefore, it is doubtful that a good diagnostics can be devised for other stars to separate the physical motion of the surface from the signatures of small planets.

The research described in this paper was carried out at the Jet Propulsion Laboratory, California Institute of Technology, under a contract with the National Aeronautics and Space Administration.

\section{REFERENCES}

Butler, R. P., et al. 2006, ApJ, 646, 505

Cumming, A. 2004, MNRAS, 354, 1165

Eggenberger, A., \& Udry, S. 2010, in Detection and Characterization of Extrasolar Planets through Doppler Spectroscopy, ed. T. Montmerle, D. Ehrenreich, \& A.-M. Lagrange (EAS Publ. Ser. 41), 27

Fischer, D. A., et al. 2008, ApJ, 675, 790

Gizon, L. 2004, Sol. Phys., 224, 217

Gizon, L., \& Rempel, M. 2008, Sol. Phys., 251, 241

Hathaway, D. H. 1987, Sol. Phys., 108, 1

Hathaway, D. H. 1992, Sol. Phys., 137, 15

Hathaway, D. H. 1996, ApJ, 460, 1027

Hathaway, D. H., et al. 1996, Science, 272, 1306

Hathaway, D. H., et al. 2000, Sol. Phys., 193, 299

Howard, R. F. 1987, Nature, 328, 667

Howard, R., et al. 1982, Sol. Phys., 83, 321

LaBonte, B. J., \& Howard, R. 1982, Sol. Phys., 80, 361

Lockwood, G. W., et al. 2007, ApJS, 171, 260

Makarov, V. V., et al. 2009, ApJ, 707, L73

Marcy, G. W., et al. 2002, ApJ, 581, 1735

McMillan, R. S., et al. 1993, ApJ, 403, 801

Meunier, N., et al. 2010, A\&A, 512, 39

Paulson, D. B., et al. 2004, AJ, 127, 1644

Saar, S. H., \& Donahue, R. A. 1997, ApJ, 485, 319

Santos, N. C., et al. 2000, A\&A, 361, 265

Santos, N. C., et al. 2010, A\&A, 511, A54

Snodgrass, H. B., \& Dailey, S. B. 1996, Sol. Phys., 163, 21

Snodgrass, H. B., \& Howard, R. 1985, Science, 228, 4702

Snodgrass, H. B., \& Ulrich, R. K. 1990, ApJ, 351, 309

Udry, S., \& Santos, N. C. 2007, ARA\&A, 45, 397

Ulrich, R. K. 2001, ApJ, 560, 466

Ulrich, R. K., \& Boyden, J. E. 2005, ApJ, 620, L123

Wright, J. T. 2004, AJ, 128, 1273 